\title{
MATISSE: A Low power front-end electronics for MAPS characterization
}

\author{
Elias Jonhatan Olave*, S. Panati, F. Cossio \\ Politecnico di Torino and INFN di Torino, Italy \\ E-mail: olave@to.infn.it, panati@to.infn.it, fcossio@to.infn.it
}

\author{
A. Rivetti, M. Da Rocha Rolo, N. Demaria \\ INFN di Torino, Italy \\ E-mail: rivetti@to.infn.it, darochareto.infn.it, \\ lino.demariadcern.ch
}

\section{Pancheri}

Università di Trento and TIFPA, Italy

E-mail: lucio.pancheri@unitn.it

\section{S. Mattiazzo, P. Giubilato, D. Pantano \\ Università di Padova and INFN di Padova, Italy \\ E-mail: piero.giubilato@unipd.it, devis.pantano@pd.infn.it, \\ serena.mattiazzo@pd.infn.it}

\begin{abstract}
In recent years Monolithic Active Pixel Sensors are becoming increasingly attractive for High Energy Physics experiments. Several R\&D activities are ongoing worldwide to improve the performance of conventional monolithic pixels in terms of speed and radiation tolerance. These improvements come both from the optimization of the substrate material and the design of the front-end electronics.

In the framework of an $R \& D$ project on $C M O S$ monolithic sensors, a versatile readout electronics has been developed. The purpose is to have a flexible system to test different sensor geometries and substrates, allowing a detailed analog characterization and the study of effects that arise in complex mixed signal chips such as digital induced noise.

The ASIC prototype, MATISSE, has been fabricated in $0.11 \mu \mathrm{m}$ CMOS technology with a die area of $2 \times 2 \mathrm{~mm}^{2}$ and a low voltage operation of $1.2 \mathrm{~V}$. Hereafter, the front-end electronics are described and the first results from the characterization are reported.
\end{abstract}

Topical Workshop on Electronics for Particle Physics

11 - 14 September 2017

Santa Cruz, California

${ }^{*}$ Speaker. 


\section{Introduction}

Silicon pixel sensors have been successfully used in many High Energy Physics experiments (HEP). Thanks to very deep sub-micron technologies it is now possible to integrate in a small area sophisticated front-end electronics to meet the challenging requirements of future particle detectors. In applications where fast readout and very high radiation tolerant are primary concerns, hybrid pixels are still the only viable solution. The much lower cost offered by monolithic CMOS sensor, is however motivating several R\&D activities to overcome the main limitation of conventional MAPS with the goal of achieving performance comparable with those of an hybrid implementations [1], [2].

The key to improve speed and radiation hardness of monolithic detectors is to have a charge collection dominated by drift. In this context, we are exploring different sensor designs and substrates materials with the aim of achieving a depletion depth of at least $30 \mu \mathrm{m}$. To allow a detailed characterization of the investigated options, a flexible front-end readout system has been developed. The chip architecture is described in section 2 and the test results are presented in section 3 .

\section{Chip architecture}
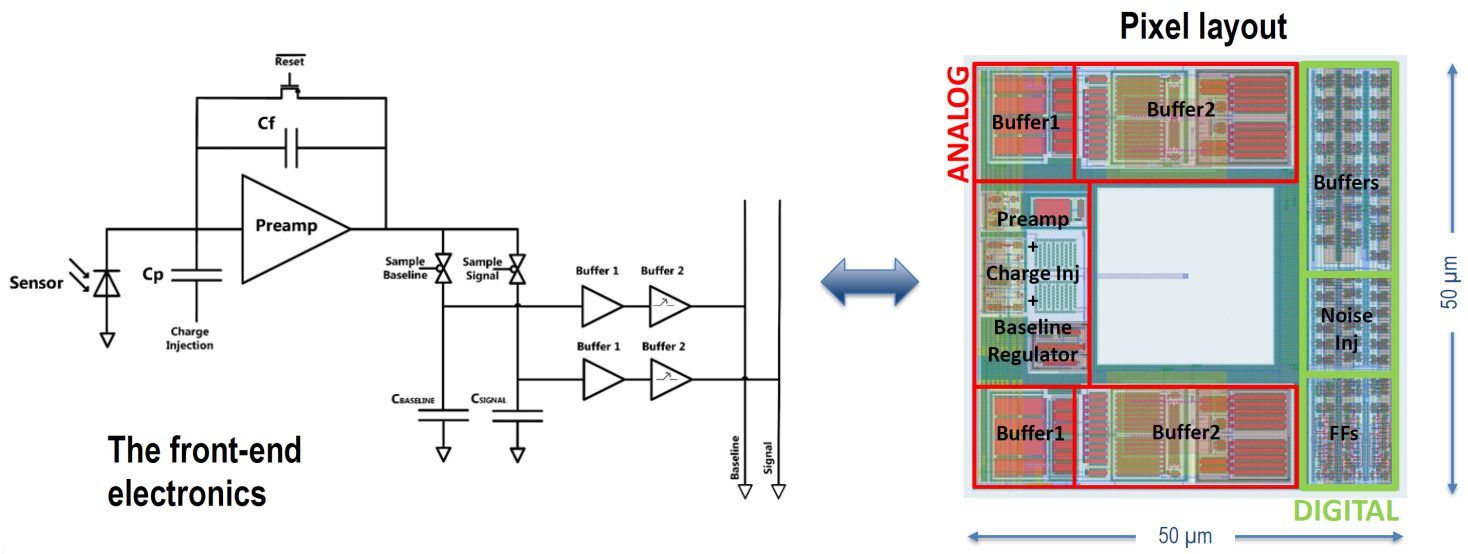

Figure 1: The analog in-pixel electronics and the layout of the pixel unit

MATISSE (Monolithic AcTIve pixel SenSor Electronics) is an ASIC prototype developed in $0.11 \mu \mathrm{m}$ CMOS technology in an area of $2 \times 2 \mathrm{~mm}^{2}$. The test chip consists of $24 \times 24$ pixel matrix divided in 4 identical groups and an End of Column logic (EoC). Each group is arranged into 6 columns of 24 pixels. The monolithic pixel unit fits a total area of $50 \times 50 \mu \mathrm{m}^{2}$ shared between a sensor of $20 \times 20 \mu \mathrm{m}^{2}$ and the in-pixel electronics as depicted in Fig.1. The sensors differ from each other for what concern the geometry of the collection electrode, the substrate type and doping. The readout electronics is common to the different sensor flavors and integrates both analog and digital circuits in proximity of the sensitive node.

The in-pixel analog readout (Fig.1) is based on a Charge Sensitive Amplifier (CSA), two local memories and four buffers used for data transmission. The CSA is based on a telescopic cascode amplifier with a feedback capacitor which fixes the conversion gain to $130 \mathrm{mV} / \mathrm{fC}$. The two 
memories are implemented with MIM capacitors to optimize the pixel size. Moreover, in the proposed design, each memory is readout by a chain formed by a low power buffer and two rail-to-rail switched op-amps which are used to send the analogue data off-chip. The whole chain has been optimized to maximize the output dynamic range up to $500 \mathrm{mV}$ maintaining a low power consumption.

The digital in-pixel logic contains three programmable flip-flops used for the readout operation and to implement features like test pulse generation and pixel masking. Moreover, the logic allows the baseline regulation which, together with the buffers wide dynamic range, makes the readout suitable for sensors of both polarities with signals in excess of $24 \mathrm{ke}^{-}$. The digital logic for the readout operation is implemented in the EoC. A chain of inverters driven by a dedicated signal allows to inject digital noise to study the sensitivity of the sensor and the very front-end in a mixed signal environment.

MATISSE supports snapshot shutter operation [4] and thus the integration time starts and ends at the same time for all the pixels of the matrix. The two signals, one at the beginning and one at the end of the integration time, are stored in the two pixel memory elements. In such a way, when some charge is collected in the sensor, it will be identified by the difference between the two stored values. For this reason both signals are sent off-chip. The readout of each sector is independently managed by the EoC logic which sequentially addresses every pixel of the sector. In this way, the matrix can be read out in less than $40 \mu \mathrm{s}$, ensuring a maximum measured voltage drop of 0.7 $\mathrm{mV}$, when a clock of $5 \mathrm{MHz}$ is used. The design supports Correlated Double Sampling (CDS) to subtract offsets in common between baseline and signal and to suppress the switching noise.

\section{Test results}

MATISSE chips were received from the foundry in April 2017 and the first results are reported here. For the characterization two dedicated PCBs have been developed: a small carrier board used to connect the ASIC to the system through wire bonding and a main board where almost all the discrete electronics is placed. Since the carrier board and main board can be easily connected, the hardware is very flexible allowing to test several chips with the same main board. The data acquisition system used for the tests is based on a software written in C++ and ROOT, a commercial FPGA and a full custom analog board with 5 channels, each with a $100 \mathrm{MS} / \mathrm{s}, 14$ bit ADC [3].

The first electrical tests were dedicated to verify the expected power consumption of $6 \mu \mathrm{W} / \mathrm{ch}$, $3.6 \mathrm{~mW}$ for the full chip during the integration period and $6.4 \mathrm{~mW}$ during the data transmission. The measurements confirm these values and suggest that all the blocks are working properly.

To study the performance of the pixel some measurements have been carried out by tuning the baseline. This operation can be done by using a voltage reference set on the main board through a dedicated trimmer and sending the proper digital signals to the ASIC. Fig. 2 shows how the baseline behaves for different voltages references. With the minimum voltage reference $(\approx 20 \mathrm{mV})$ the baseline reaches its maximum $(700 \mathrm{mV})$ and then for higher voltage references it decreases with a linear response down to $\approx 320 \mathrm{mV}$. The measurement shows that the expected operation range for the analog in-pixel electronics is covered with a good linearity.

As a first check of the combined sensor and electronics performance, a measurement with a

${ }^{55} \mathrm{Fe}$ radioactive source has been done. In this measurement the integration time and the baseline 


\section{BASELINE REGULATION}

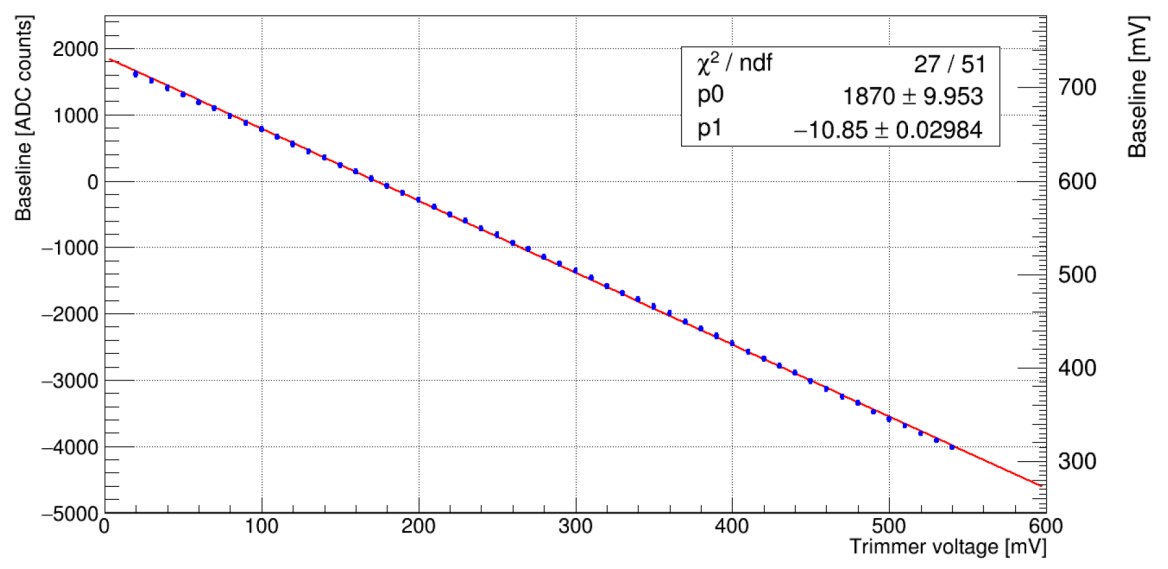

Figure 2: Regulation of the baseline for voltage references in the range of $20 \mathrm{mV}$ and $550 \mathrm{mV}$

have been set to $5 \mu \mathrm{s}$ and $450 \mathrm{mV}$ respectively. Fig. 3 shows the reconstruction spectrum where the main peak at $5.9 \mathrm{keV}$ is clearly seen. From this measurement it follows that the analog gain of the full readout chain is $\approx 117 \mathrm{mV} / \mathrm{fC}$. This value is in fair agreement with the one expected from computer simulations $(\approx 130 \mathrm{mV} / \mathrm{fC})$.

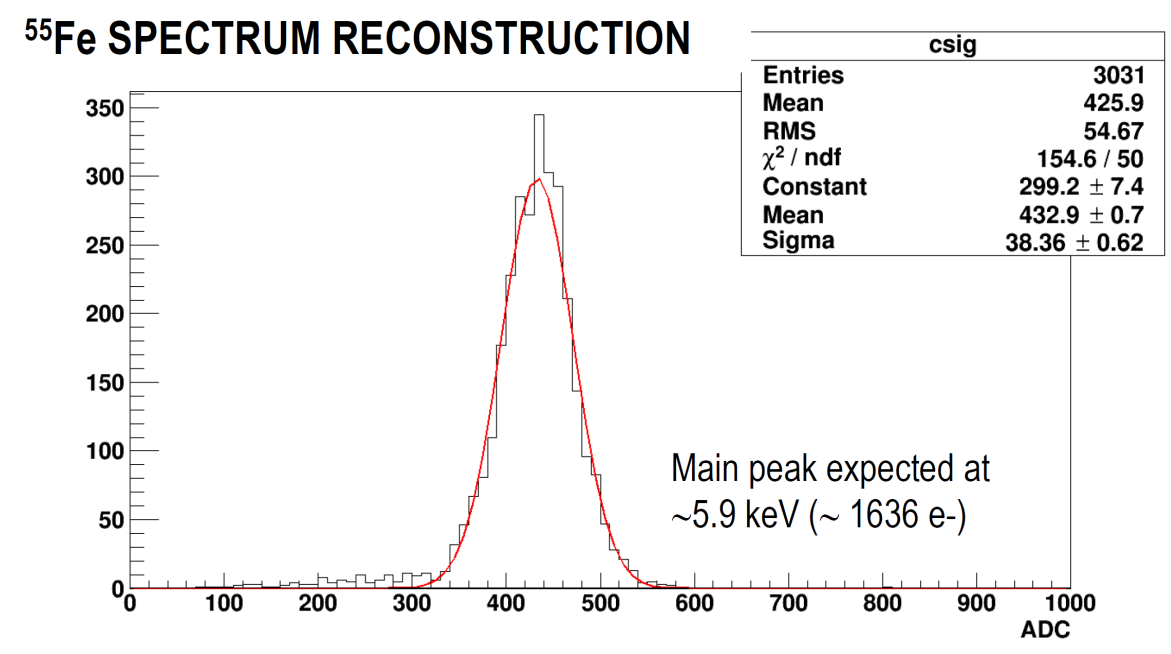

Figure 3: ${ }^{55} \mathrm{Fe}$ spectrum reconstruction

MATISSE has been designed to keep the noise at the preamplifier output below $1 \mathrm{mV}$ rms. The full chain has been analyzed to estimate the noise of each block. In Table 1 the contributions from the DAQ box, main board, carrier board and finally from the ASIC itself are summarized. The noise up to the carrier board is very low $(110 \mu \mathrm{V})$ therefore, this confirms that the DAQ system is adequate for noise measurements.

Fig. 4 shows the noise of all pixels in a matrix. The mean noise value is $1.1 \mathrm{mV}$ which corresponds to $58 \mathrm{e}^{-}$rms. 


\begin{tabular}{lll}
\hline DEVICE & NOISE [ADC] & NOISE [mV] \\
\hline DAQ box & 0.2 & 0.02 \\
\hline DAQ + Main Board & 0.2 & 0.02 \\
\hline DAQ + Main Board + Carrier board & 1.6 & 0.11 \\
\hline DAQ + Main Board + Carrier board + ASIC & 15 & 1.0 \\
\hline
\end{tabular}

Table 1: Noise measurement in the full readout chain.

NOISE DISTRIBUTION

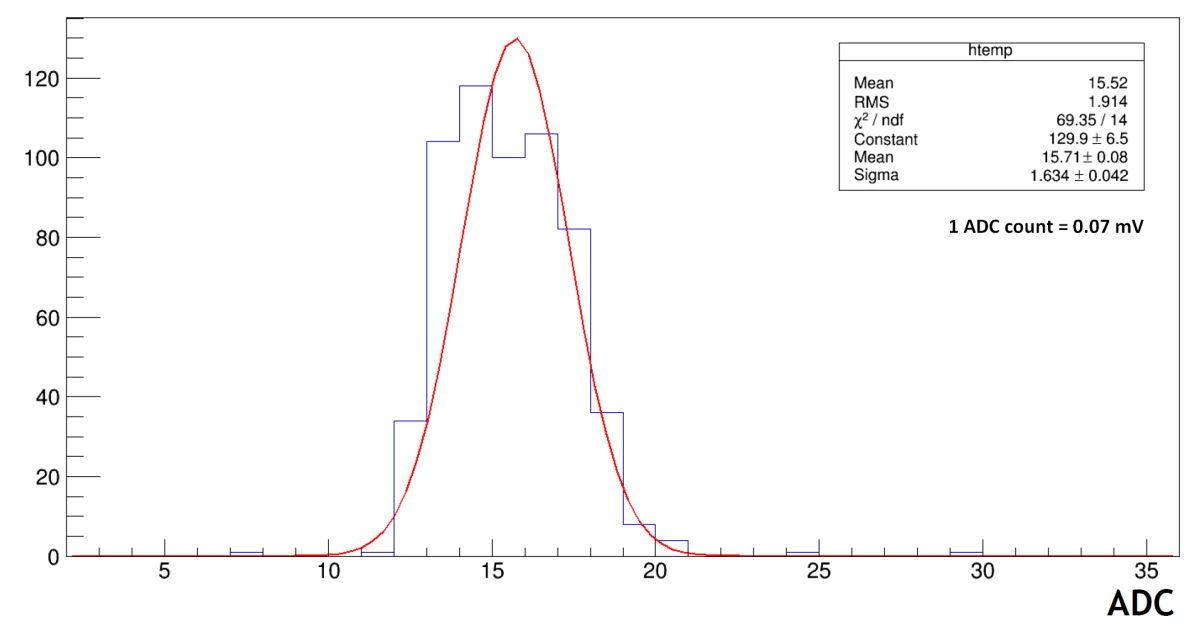

Figure 4: Noise distribution of the full matrix

\section{Conclusions}

A front-end electronics for the characterization of different CMOS sensors implemented in $0.11 \mu \mathrm{m}$ CMOS technology has been developed. The circuit is intended to allow a detail characterization of the sensor performance through analog readout and to explore issues arising from the simultaneous integration in the pixel of fast digital gates. First results show that the readout electronics is fully functional. The baseline can be adjusted in a wide range making the front-end adequate for sensors of either polarity. Preliminary measurements with a ${ }^{55} \mathrm{Fe}$ source show a gain of $117 \mathrm{mV} / \mathrm{fC}$ and a noise of $58 \mathrm{e}^{-}$rms. The study of the digital noise injected by the dedicated test structures and the characterization of matrices containing different sensor optimization is in progress.

\section{References}

[1] R. Turchetta et al.,A monolithic active pixel sensor for charged particle tracking and imaging using standard VLSI CMOS technology. NIM A 458, 677-689, 2001.

[2] W. Snoeys, Monolithic pixel detectors for high energy physics. NIM A 731, 125-130, 2003.

[3] M. Battaglia, D. Bisello, D. Contarato, P. Giubilato, D. Pantano, M. Tessaro, "A DAQ system for pixel detectors R\&D"'. Nuclear Instruments and Methods in Physics Research A, Elseiver, A 611, 2009

[4] M. Innocent, General introduction to CMOS image sensors, Cypress Semiconductor, Belgium. 